\title{
Carl Schmitt, lecteur de Tocqueville
}

La démocratie en question

\section{Anaïs Camus et Tristan Storme}

\section{(2) OpenEdition}

\section{Journals}

Édition électronique

URL : http://journals.openedition.org/ress/785

DOI : $10.4000 /$ ress.785

ISSN : 1663-4446

Éditeur

Librairie Droz

\section{Édition imprimée}

Date de publication : 15 octobre 2011

Pagination : 7-35

ISBN : 978-2-600-01551-6

ISSN : 0048-8046

\section{Référence électronique}

Anaïs Camus et Tristan Storme, "Carl Schmitt, lecteur de Tocqueville», Revue européenne des sciences sociales [En ligne], 49-1 | 2011, mis en ligne le 01 janvier 2015, consulté le 06 mai 2019. URL : http:// journals.openedition.org/ress/785; DOI : 10.4000/ress.785 


\title{
CARL SCHMITT, LECTEUR DE TOCQUEVILLE
}

\section{LA DÉMOCRATIE EN QUESTION}

\author{
ANAIIS CAMUS \& TRISTAN STORME \\ Université libre de Bruxelles \\ anais.camus@ulb.ac.be/trstorme@ulb.ac.be
}

\begin{abstract}
Résumé. Alexis de Tocqueville apparaît comme le symbole d'un renouveau de la pensée libérale française, tandis que Carl Schmitt est souvent considéré comme l'adversaire le plus brillant du libéralisme. Chercher à les rapprocher peut donc sembler contre-intuitif. Cependant, une étude approfondie des écrits de Schmitt montre qu'il éprouvait une véritable admiration pour l'historien français. Cet article propose de revenir sur ce qui semble constituer une interprétation apparentée du phénomène démocratique et d'une fin possible du politique. Les auteurs y défendront l'idée selon laquelle, tout en partant de questions fort divergentes, voire opposées, ces deux penseurs témoignent d'une profonde convergence problématique. Outre l'expression d'une crainte commune à l'égard de l'élargissement des frontières nationales, de l'individualisme et de la souveraineté populaire, c'est plus généralement, selon les auteurs, la portée théologico-politique de leurs pensées respectives qui justifie un tel rapprochement.
\end{abstract}

Mot-clés: Carl Schmitt, Alexis de Tocqueville, démocratie, dépolitisations, critique de l'individualisme, souveraineté populaire

\begin{abstract}
Tocqueville can easily be seen as the symbol of the French liberal thought whereas Schmitt is considered as the leading opponent of the liberal doctrine. Trying to reconcile their theories might thus seem counter-intuitive. However, delving into Schmitt's work reveals that the German thinker admired his French counterpart. This article offers a first glance into what appears as a somehow connected interpretation of the democratic phenomenon in relation to their fear of possible depoliticisations. The authors will argue that, while they elaborate on distinct and almost contradictory questions, the historian and the jurist show a deep convergence of problématique. If their reservations concerning the enlargement of national borders, individualism and popular sovereignty reinforce this intuition, these similarities will be, in conclusion, traced back to a common use of a theological-political framework.
\end{abstract}

Keywords: Carl Schmitt, Alexis de Tocqueville, democracy, depoliticisations, critique of individualism, popular sovereignty 


\section{INTRODUCTION}

Durant les années 1970, Alexis de Tocqueville et Carl Schmitt sont apparus comme les successeurs potentiels du vide philosophique laissé par la chute de la pensée marxiste' ${ }^{1}$ Toutefois, en apparence, ils s'opposent radicalement. Tocqueville serait le symbole d'un renouveau de la pensée libérale en France, tandis que Schmitt est souvent considéré comme l'adversaire le plus brillant du libéralisme. Chercher à les rapprocher peut donc sembler contre-intuitif, même si certains commentateurs ont pu pointer du doigt la pertinence d'un tel rapprochement ${ }^{2}$. Daniel Lindenberg (2002, p. 58-60) a soutenu, dans un ouvrage très controversé, que de nombreux néo-tocquevilliens français se seraient épris des réflexions de Carl Schmitt. D’autres ont cru observer que certains auteurs, qui passent pour avoir redonné son importance, en France, à l'historien du XIX ${ }^{\mathrm{e}}$ siècle, se dissimuleraient derrière un Tocqueville cachant, en réalité, l'ombre dense et tenace de la pensée du publiciste de Weimar. Ainsi, Serge Audier (2009, p.I83) estime-t-il que les craintes de Pierre Manent face au déclin présumé de l'État-nation lui sont inspirées par Carl Schmitt, bien qu'il préfère mobiliser Tocqueville.

Mais il faut reconnaître qu'en dehors d'un court essai: «Historiographia in nuce: Alexis de Tocqueville», rédigé en 1946 et repris en 1950 dans Ex Captivitate Salus, Schmitt n'a pas écrit grand-chose sur Tocqueville. Pourtant, dans les écrits où il le mentionne, le juriste se montre particulièrement élogieux envers lui. Il le présente comme «le plus grand historien du XIx siècle» (Schmitt, 2003 [1950], p. I36), auquel il ne manque pas de s'identifier. En réalité, Schmitt s'est souvent référé aux textes de Tocqueville, la plupart du temps sous forme de commentaires furtifs et sommaires, parfois placés en note. Malgré les attaques schmittiennes répétées à l'encontre des théoriciens libéraux, le penseur français semble bénéficier d'un traitement de faveur - ce qui eut de quoi étonner certains commentateurs33. Günter Rohrmoser (1998, p. I48-I49), qui rencontra Carl Schmitt à

I Voir, par exemple, Audier (2004) pour Tocqueville, et Agnoli et Brückner (1968) concernant Schmitt.

2 Voir, parmi d'autres, Scheuerman (1999, p. 43-44, p. 86-87, p. 98-99).

3 Voir, en particulier, Balakrishnan (2006 [2000]). 
Münster, affirma que l'auteur d'Ex Captivitate Salus «avait l'intention d'écrire un plus gros ouvrage sur Tocqueville» que le petit texte rédigé en 1946. Piet Tommissen (200I, p. I05-I09) fit paraître en 200 I une retranscription de notes sténographiées du juriste entièrement dévolues à la pensée de Tocqueville. Les «C.S.s Tocqueville-Notizen», certes peu fournies, viennent néanmoins aider à comprendre l'admiration que Schmitt vouait à l'historien français, dont il possédait, paraît-il, un portrait qu’il n'était pas rare, au début des années I980, d'apercevoir sur le pupitre de son bureau ${ }^{4}$. L'anecdote pourrait-elle indiquer une forme de proximité insoupçonnée entre les deux auteurs? Si certains commentateurs ont pu imaginer que, derrière ses usages répétés de la pensée de Tocqueville, un philosophe comme Pierre Manent tendrait à exhumer in fine un argumentaire d'inspiration schmittienne, une proximité problématique rapproche-t-elle peut-être ces deux penseurs qu’un siècle sépare?

La confrontation des deux auteurs ne visera en aucun cas à faire la revue de leurs divergences et convergences doctrinales. Pareil exercice serait périlleux en raison de systématiques conceptuelles pratiquement incompatibles. Ces penseurs partent de questions princeps très différentes: alors que Tocqueville se demande comment préserver la liberté dans un contexte d'égalité, Schmitt s'interroge quant aux moyens de maintenir le politique et ses conditions d'existence dans un contexte de mondialisation libérale. Néanmoins, nous chercherons à montrer que, tout en partant de questions fort divergentes, voire opposées, qui se développent dans des architectoniques dissemblables, l'historien et le juriste témoignent d'une profonde convergence problématique : celle des limites politiques de la démocratie et, en dernière instance, celle de la menace des «dépolitisations » à leurs époques respectives, inquiétude autour de laquelle tournent leurs pensées sans jamais pour autant s’y réduire.

Dans une première partie, nous approcherons les observations respectives des deux auteurs en ce qui concerne la structure de la société démocratique. L'unité politique, les dangers de la diversité, l'égalité et l’homogénéité jouent un rôle conceptuel fondamental dans leurs préhensions du phénomène démocra- 
tique; ce qui vient soutenir l'idée d'une proximité intellectuelle, à ceci près - et ce n'est pas rien - que Schmitt s'entête à évincer la variable «liberté » de l'équation démocratique tocquevillienne. Nous examinerons ensuite la parenté de leurs mises en garde contre les risques inhérents au développement de la démocratie, ainsi que les orientations normatives qui en découlent. La seconde partie de cet article se concentrera sur leurs craintes qui ont trait à l'élargissement des frontières nationales. Le juriste allemand était fasciné par le «grand pronostic» de l'historien français sur le futur démocratique de l'humanité : il était convaincu que Tocqueville encourageait la France à retrouver sa «vie nationale». Une troisième partie étudiera leurs critiques respectives de l'individualisme et de la souveraineté populaire révélatrices de la manière dont ils envisagent la nature du peuple, des individus qui le composent et de la liberté dont ils pourraient jouir. En conclusion, l'aspect théologico-politique de leurs pensées viendra éclairer ce qui les rapproche. On peut à tout le moins penser que cet aspect fournit un cadre théorique logiquement antérieur aux conclusions politiques comparables de nos deux auteurs. Un tel cadre indiquerait certains fondements partagés, à même de sous-tendre l'intégration de certaines parts de la théorie tocquevillienne dans les considérations de Schmitt relatives à la démocratie.

\section{LA STRUCTURE CONCEPTUELLE DE LA DÉMOCRATIE}

Si l'on cherche à saisir la définition schmittienne du politique, téléologiquement liée au pôle étatique-démocratique dans les années $1920^{5}$, on ne saurait se concentrer uniquement sur l'hostilité. L'ennemi, dont le surgissement compose certes le critère spécifique du politique, «ne peut pas être conçu en dehors de la notion d’amitié, par laquelle les individus sont amenés à constituer des “collectivités” » (Norris, 1998, p. 73). Schmitt (2004 [1932], p. 77) affirme que tout est à même de devenir politique, dans la mesure où la notion peut éclore de n’importe quel prétexte de regroupement, pour autant que ce critère

5 Bien que Schmitt distingue logiquement (ou conceptuellement) l'État du politique dans la première phrase de son essai de 1927, Derrida (1994, p. 140) considère que la forme étatique du politique lui «sert souvent de télos ou de fil conducteur pour sa définition du politique». 
corresponde au degré d'association le plus intense ${ }^{6}$. L’amitié initiale a donc pour condition négative fondamentale la réduction des pluralismes au bénéfice d'un seul et unique critère d'appartenance. Le peuple, rassemblé sous l'impulsion d'un tel critère d'association, se forge en une unité nationale cohérente, chaque nation possédant «son propre concept de nation» et trouvant «en elle-même, et non chez les autres, les traits constitutifs de la nationalité» (Schmitt, 2004a, p. I38). Aux yeux du juriste allemand, le peuple est toujours le peuple rassemblé, enclin à être représenté. Il use de l'expression «unité politique » lorsqu’il évoque l’État ou la base nationale nécessaire à sa bonne tenue: «En réalité, il n’existe pas de société ou d'association politique, il n'y a qu’une unité politique [politische Einheit], une communauté politique» (Schmitt, 2004 [1932], p. 83-84).

Cette idée d'unité politique se retrouve également chez Tocqueville. En effet, il lie le bon gouvernement et, dans ce cas-ci, le bon gouvernement démocratique à une unité sociale et politique prédéfinie (Tocqueville, 1977, p. II3). En d'autres termes, la centralisation qui a accompagné l'avènement de la démocratie a créé l'unité politique nécessaire au développement de cette même démocratie. Il ajoute par ailleurs qu'une nation a besoin pour prospérer d'une centralisation gouvernementale suffisamment forte. Aussi - et c'est ici que la comparaison devient intéressante -, il reconnaît que cette unité politique doit se construire autour de valeurs communes, à l'image de l'identité nationale. Ce sentiment national se baserait non seulement sur une habitude du vivre-ensemble, mais aussi sur des opinions, des impressions ou des pensées partagées (Tocqueville, I986 [1835], p. 543). À l'inverse, une diversité nationale trop forte représenterait un risque pour la démocratie, car il deviendrait trop pénible de se soumettre au privilège de la majorité (ibid., p. I50). En bon penseur libéral, Tocqueville laisse néanmoins la porte ouverte à d’autres associations dans l'association, ou à d'autres nations au sein de la nation (ibid., p. 289). Contrairement à ce qu'on peut lire chez Schmitt, la nation se compose alors de plusieurs entités distinctes dont l'État-nation n'est que le degré idéal nécessaire au bon gouvernement à

6 L'amitié identitaire ne prend pas sa source dans un domaine spécifique ou dans un type particulier de rapports humains, mais dans une valeur intensive - «le plus haut degré d'intensité»-, susceptible de qualifier toute relation collective, aussi divers que puissent être les motifs initiaux qui la déterminent. 
un moment donné. Toutefois, cette croyance commune en la nécessité d’une identité substantielle qui assurerait le maintien d'une société politique n’est pas concluante en tant que telle puisqu'elle n'est pas spécifique à ces deux auteurs et ne nous permet pas de qualifier la singularité de leurs pensées respectives. Il nous est donc indispensable d’approfondir cette analyse.

Chez l'un comme chez l'autre, l'unité politique précède logiquement l'individu : elle est une identité hypostasiée, le réquisit d’une lecture du phénomène démocratique qui, pour sa part, dépend étroitement du cercle formé par les notions d'«homogénéité» et d'«égalité». Cette condition préalable de la démocratie s'associe également à une vision partagée du phénomène démocratique en tant que phénomène social qui s’immisce dans toutes les actions humaines. Ces deux constats qui découlent, pour ainsi dire, de la notion de communauté figurent à la souche de leurs théories de la démocratie et les amènent à des conclusions comparables.

Carl Schmitt présente nommément son concept d’État en tant qu'État « démocratique». Celui-ci repose sur un principe particulier d'égalité, annoncé comme partie prenante de l'imaginaire démocratique: «L'égalité (Gleichheit) démocratique est essentiellement homogénéité (Gleichartigkeit), l'homogénéité d'un peuple. La notion essentielle de la démocratie, c'est le peuple et non l'humanité » (Schmitt, 1993 [1928], p. 37I). Dans les réflexions du juriste, l’homogénéité se laisse ainsi déduire à partir de l'égalité. L'homogénéité apparaît comme la condition première d'une unité nationale effective - le peuple devient nation dès lors qu'il prend pleinement conscience de sa spécificité politique et de sa volonté d'exister politiquement. Cette notion d'égalité «substantielle» entre tous les membres du corps ou de la communauté politique consacre, en premier lieu, l'absence de différence qualitative entre les gouvernants et les gouvernés. Ceci implique, dans un second temps, que l'égalité conçue comme homogénéité nationale ne saurait être absolue; la démocratie n'ignore aucunement le concept d'étranger. Il n’y a que les nationaux qui sont «égaux» au sein de l'État démocratique; ils sont les seuls à disposer de droits politiques, étant donné que la démocratie s’adosse au principe d’appartenance à une nation précise et qu'elle refuse l'indistinction entre les hommes. Même si une minorité nationale détient 
certains droits, «elle n'est pas censée avoir des droits politiques en tant que nation à l'égard de la nation dominante » (ibid., p. 369). Les non-nationaux, en ce sens, ne sont pas appelés à participer aux affaires publiques. Bien plus, afin de conforter l'homogénéité du peuple, l'effort démocratique passerait nécessairement par «le contrôle de l'immigration étrangère et le rejet des éléments étrangers indésirables» (ibid, p. 370), entendons par l'éviction de «l'ennemi intérieur ».

La démocratie tocquevillienne se présente comme moins exclusive et plus ouverte aux différences que celle de Schmitt. Cependant, le penseur français considérait lui aussi qu'il existait un cercle de dépendance entre l'égalité et l'homogénéité. Si d’après Schmitt l'égalité s’affiche à travers l'homogénéité, Tocqueville regarde, quant à lui, cette dernière comme une conséquence de l'égalité, originellement perçue comme l'égalité des conditions sociales. La démocratie impliquerait, en effet, une hausse de l'individualisme, et alors que les citoyens se veulent de plus en plus particuliers, ils n’auraient de cesse en réalité de devenir similaires. Les individus donnent à un ensemble abstrait une puissance intellectuelle, voire physique, qu'ils ne donnent pas aux parties de cet ensemble prises indépendamment - et ce au travers du concept d'opinion publique (Tocqueville, I98 I [I840], p. i7). Dès lors, on assisterait à une délégation croissante du pouvoir identitaire entre les mains du gouvernement central, ainsi qu'à un approfondissement de la centralisation (ibid, p. 362). Cette centralisation serait elle-même responsable de l'homogénéisation des individus, tout comme d’un approfondissement de la perception d'égalité qui les soude les uns aux autres. Bien qu'il n'utilise que très rarement le terme d'«homogénéité», l’historien français décrit un processus que ses commentateurs regardent comme un processus d'homogénéisation ou du moins d'uniformité croissante?

Probablement au fait de cette ressemblance, Schmitt considérait que Tocqueville avait également pris conscience de ce que les révolutions mettaient en danger l'homogénéité du peuple, l’aspect égalitaire de la démocratie. En effet, «la situation que Tocqueville avait si bien saisie en i830 [...] [révéla] son vrai 
visage, celui de la guerre civile» (Doremus dans Schmitt, 2003 [1950], p. 212), moins de vingt ans plus tard. Le juriste allemand fait ici référence aux Souvenirs de Tocqueville. Alors que l'historien livre son témoignage sur les événements qui ont secoué la société française en 1848 , il concède avec clarté qu’il «ne voulait pas de révolution» (Tocqueville, 1999 [1964], p. I23). Contrairement à ce quavance Schmitt, cette déclaration d’apparence anti-révolutionnaire est néanmoins nuancée en d’autres endroits de l'œuvre tocquevillienne. Selon le français, il n’y a pas lieu de craindre les révolutions, qui, la plupart du temps, amènent plus de bien que de mal. Loin d'avoir peur pour l'homogénéité du peuple, lorsqu'il critique la révolution dans ses Souvenirs, c'est pour la liberté que Tocqueville s'inquiète: «Je savais que, si une grande révolution peut fonder la liberté dans un pays, plusieurs révolutions qui se succèdent y rendent pour très longtemps toute liberté régulière impossible ${ }^{8} \gg$ (ibid., p. 88).

La notion d'ennemi intérieur n’a pas sa place dans la théorie de l'historien français, qui n’a jamais considéré les minorités nationales comme des menaces pour la démocratie. S’il prévoit que l'homogénéité puisse devenir la norme (Jacques, 2005, p. 380) et que les conflits internes disparaîtront probablement avec le développement de la démocratie (Tocqueville, 198 I [1840], p. 88), la présence de divergences et de minorités nationales demeure une nécessité quoiqu'une hétérogénéité trop prononcée de la population risque de mener à la fin du jeu démocratique. Il est favorable à tous les débats, aussi antagonistes soient-ils, tant que ceux-ci permettent aux idées de circuler et aux citoyens de prendre part à la vie politique; mais, dans un même temps, force est de reconnaître que, pour lui, cette participation requiert une certaine unité politique. Cette ambivalence à l'égard de l'homogénéité peut expliquer pourquoi Schmitt est capable de recourir à une part des observations tocquevilliennes, qu'il intègre dans sa propre définition de la démocratie.

8 Tocqueville ajoute ailleurs que si les individus se coupent des émotions publiques intenses telles que les révolutions, ils risquent de considérer «toute théorie nouvelle comme un péril, toute innovation comme un trouble fâcheux» (Tocqueville, I98I [1840], p. 323-324). 
On pourrait penser que leur utilisation conjointe du terme d'«homogénéité » ne serait qu’une affaire d’homonymie. Nous estimons néanmoins qu'il est sérieusement possible d'envisager un réel rapprochement conceptuel entre leurs perceptions respectives de l'homogénéité nationale. Tandis que ce concept est bien connu des commentateurs de Schmitt, cela semble moins évident concernant Tocqueville. On peut cependant défendre qu'une certaine homogénéité nationale, qui résulte d’une égalité croissante, s’avère nécessaire au développement de la liberté tocquevillienne, ne serait-ce que pour assurer l'unité politique. L'historien avance que, «[p]our qu'une confédération subsiste longtemps, il n’est pas moins nécessaire qu’il y ait homogénéité dans la civilisation que dans les besoins des divers peuples qui la composent » (Tocqueville, 1986 [1835], p. 258). Le penseur français reconnaît explicitement le caractère indispensable d'une homogénéité spécifique pour assurer la survie d'une confédération, ce qui est a fortiori vrai pour les nations plus centralisées. Cette ambivalence, ou pourrait-on dire: cette contradiction, au sujet du phénomène national dans la théorie d’Alexis de Tocqueville a poussé certains auteurs à le qualifier de «nationaliste »'. Tandis que l'aristocrate français tente de maintenir un certain degré d'unité au sein de la population dans l'optique de protéger cette liberté politique, «[u]n État nationalement homogène devient alors le cas normal», selon le juriste allemand (i993 [1928], p. 369). L’homogénéité nationale représente la condition préalable de l’efficience de l'État démocratique ${ }^{10}$. Car, dans la pensée de Carl Schmitt: «[P]our que le peuple $[. .$.$] advienne à l'existence politique, il faut une certaine identité, une$ certaine homogénéité en son sein » (Schmitt, 1990, p. 34). On comprend que la configuration démocratique de l'État devrait supposer, d'une part, une nation homogène et signifier, d'autre part, la lutte perpétuelle en vue du maintien et de la préservation de l'homogénéité initiale ${ }^{11}$. La démocratie exprime donc la voluntas du peuple - un sentiment fort d'appartenance, un critère extrême d’asso-

9 À ce sujet, consulter Pitts (2005).

10 «Toute démocratie suppose une homogénéité totale du peuple» (Schmitt, 1990, p. 37).

II Scheuerman (1995, p. 139) condamne, parmi d'autres, cette conception schmittienne de l'État démocratique. 
ciation -, qu'elle se doit de «corriger » en permanence, ou du moins d'entretenir et de «former», par l'intermédiaire de la raison d’État - une ratio dont seul le pouvoir est capable ${ }^{12}$. Ce qui semble constituer le fondement central de la démocratie schmittienne est pour Tocqueville un développement potentiellement dangereux, quoique nécessaire.

Schmitt voyait dans le découplage analytique entre nation et démocratie - entre souveraineté nationale-étatique et souveraineté populaire - l'annonce de dépolitisations à venir. Dans La Raison des Nations, Pierre Manent s'insurge contre le fait que nous serions aujourd'hui sortis de la période «tocquevillienne» de la démocratie (Manent, 2006, p. 25). Ce souci tocquevillien de l'homogénéité nationale, pas très éloigné de celui de Schmitt, pourrait composer un indice de l'existence de passerelles ou de carrefours communs aux problématiques des deux auteurs.

\section{PENSER LES FRONTIÈRES EN DES TEMPS DÉMOCRATIQUES}

Contre l'héritage de la Révolution française?

$\mathrm{Si}$, d’après le juriste allemand, Tocqueville siège au faîte de la caste des historiens, c'est principalement en raison du «grand pronostic qui se trouve à la fin du premier tome de sa Démocratie en Amérique» (Schmitt, 2003 [1950], p. 137) et qu'il aurait formulé avec génie, alors que le grand Hegel lui-même - dont on connaît l'importance dans la pensée de Carl Schmitt - mourut sans être parvenu à prédire ce phénomène déterminant pour l'histoire politique mondiale, encore moins à nommer les futurs porteurs de ce développement nouveau. En avance sur son temps, l'historien français aurait prévu la longue marche du globe vers une humanité centralisée et démocratisée. Non content d’avoir prédit cette tendance irrésistible «vers la centralisation et la démocratisation», Tocqueville aurait ensuite désigné les puissances historiques concrètes capables de soutenir ce développement inédit et de l'imposer : l'Amérique et la Russie (ibid) ${ }^{13}$. Le penseur

12 À ce propos, voir Tertulian (1996, p. 145).

13 Deux ans auparavant, Schmitt (1980a, p. 88-89) écrivait que La Démocratie en Amérique «a établi ce pronostic qu'une inévitable démocratisation et centralisation de l'humanité trouverait son accomplissement en Russie et aux États-Unis d'Amérique». 
allemand est formel: Tocqueville fut le premier auteur à entrevoir la chute de l'Europe de son piédestal et l'éclosion des deux nouvelles puissances mondiales.

Carl Schmitt relève qu'en dépit de sa popularité, cette intuition n’a été que très peu prise au sérieux par les intellectuels d’Europe (Schmitt, I950a, p. 89). Après 1945, le juriste allemand soutient que l'opposition entre le libéralisme américain et le communisme soviétique ne serait qu'une opposition de façade, à tel point qu'il comprend la guerre froide comme «une pax américano-soviétique». Dans la confrontation de l'après-guerre, Schmitt ne perçoit aucun Nomos, aucun ordre spatialisé. Ces superpuissances sont, pour ainsi dire, inaptes à remplir la fonction tenue précédemment par les États - à savoir, au bout du compte, l'inférence d'un «plurivers». Pour Schmitt, on ne saurait nier que les hommes se répartissent suivant le syntagme déterminant de l'ami et de l'ennemi, façonnant le globe en une pluralité, en un pluriversum d’États; ce qui signifie, en dernière instance, que l'humanité n'est nullement disposée à composer un critère d'association rigoureux (Schmitt, 2004 [1932], p. 95) Afin d'unifier le monde, les États-Unis embrasseraient une nouvelle idéologie humanitaire qui correspondrait largement à l'instrumentalisation du concept d'humanité, particulièrement utile aux expansions impérialistes. Quiconque invoquerait l'humanité dans l'optique de combattre ses opposants, avec une morale universelle en front de bandière, refoulerait cet adversaire, d'un même geste, en dehors de la loi et de l'espèce humaine. La guerre révolutionnaire russe qui mine les gouvernements étrangers et tente de subvertir l'ordre social des autres pays ne serait pas bien éloignée de cette volonté d'unification du monde qui n’a que faire des souverainetés étatiques, corroborant dès lors la géniale intuition du grand historien français.

Alexis de Tocqueville critique, lui aussi, l'idée d'un État mondial. Il sait que le développement de semblable État universel est possible et qu'il sera le résultat d'une démocratie qui se répand de plus en en plus (Tocqueville, I98I [I840], p. 346, n. 2). «Le Moyen Âge était une époque de fractionnement [...]. De nos jours, un mouvement contraire se fait sentir, les peuples semblent marcher vers l'unité» (Tocqueville, I986 [1835], p. 596-597). Comme Schmitt un siècle plus tard, il craint toutefois pareille évolution. Il nous apprend qu'il est «permis 
de dire d'une manière générale que rien n'est si contraire au bien-être et à la liberté des hommes que les grands empires», puisque «le législateur est obligé de donner aux lois un caractère uniforme que ne comporte pas la diversité des lieux et des mœurs » (ibid., p. 247-249). L'Empire mettrait en danger la liberté et la diversité de la société mondiale. Tocqueville met néanmoins en exergue trois facteurs qui devraient naturellement empêcher la réalisation de cette homogénéisation de la population: le nationalisme (Tocqueville, 1973, p. I08), la survie de certaines caractéristiques identitaires (ibid., p. 39) et la faiblesse croissante de toutes les nations (Tocqueville, 1986 [1835], p. I58); il sappuie ici sur une logique d'après laquelle «dans l'immense complication des lois humaines il arrive quelquefois que l'extrême liberté corrige les abus de la liberté, et que l'extrême démocratie prévient les dangers de la démocratie» (ibid., p. 295). À l'instar de ce qui occupe le cœur des réflexions de Schmitt après 1945, l'humanité n’est pas, pour Tocqueville, un critère d'association souhaitable. Non pas parce que l'humanité ne serait pas politique par nature, mais parce qu'elle mènerait à une fin du politique de par l'homogénéisation excessive de ses populations.

Schmitt ne semble pas être conscient de cette croyance tocquevillienne en certains facteurs capables de freiner l'unification de l'humanité. Serait-ce une erreur d'interprétation, volontaire ou non, de la part du juriste allemand? Le fameux pronostic s'énonce de la sorte:

Il y a aujourd'hui sur la terre deux grands peuples, qui partis de points différents, semblent s'avancer vers le même but: ce sont les Russes et les AngloAméricains [...]. L’un a pour principal moyen d'action la liberté; l'autre, la servitude. Leur point de départ est différent, leurs voies sont diverses; néanmoins chacun d'eux semble appelé par un dessein secret de la Providence à tenir un jour dans ses mains les destinées de la moitié du monde. (Ibid., p. 598.)

Alors que Schmitt concentre son argumentation sur la dernière partie du développement de Tocqueville, il est clair que le théoricien français paraît moins engagé que ce que ne prétend l'auteur allemand. Ce dernier s'appuie sur ce passage pour expliquer un événement qui s'est produit bien longtemps après la mort de l'historien français : l'interprétation de Schmitt est complètement biaisée par la guerre froide. Et même si Tocqueville envisage à l'occasion 
la possibilité d'une unification d'échelle mondiale, cet extrait ne serait pas le paragraphe le plus pertinent pour l'illustrer.

Piet Tommissen est d'avis qu'il est très compliqué de savoir quand, précisément, Carl Schmitt a commencé à se passionner pour l'œuvre d’Alexis de Tocqueville. Il émet toutefois l'hypothèse que l'estime et l'intérêt du juriste ont pu naître lors de la lecture de l'Histoire de la science politique dans ses rapports avec la morale de Paul Janet. Celui-ci considérait que Tocqueville comptait parmi les esprits libres «dont le nom a toujours été grandissant et l'importance de plus en plus appréciée, depuis que les faits ont confirmé quelques-unes de ses plus graves prévisions » (Janet, I887, p. 736). Pourtant, les descriptions que ce dernier a livrées au sujet de l’Amérique dans son opus magnum, en particulier de la diplomatie, ne seraient non seulement plus valables aujourd'hui sur bien des points, mais n’auraient pour ainsi dire jamais été vraies. Dans ses notes sténographiées, Schmitt soutient qu'«il ne subsiste rien en réalité» des constats sociologiques de Tocqueville, qui ne sont que l'illustration des préjugés qui l’habitaient (dans Tommissen, 200I, p. 107). «Quelque chose était correct en se référant à l'Amérique, mais pas en démocratie; une autre en se référant à la diplomatie en générale, mais pas en Amérique» (ibid., p. I08). En dépit de cela, Schmitt regardait tout de même l'historien comme un «bon observateur» dont le juste sens rendait sans importance l'inexactitude des descriptions sociologiques pensées a priori. C’est parce qu’il avait réussi à se détacher des visions marquées par l'époque qu’il était parvenu à prédire la rencontre russo-américaine et l'unification de l'humanité à partir des valeurs démocratiques - c'était bien là l'essentiel. Le sens tocquevillien de l'observation sávérait bien plus grandiose que toutes les grandes prédictions «abstraites, très attachées au temps, sans grande vision» (ibid., p. 107) de ses contemporains, dès lors qu'il sagissait de se prononcer sur le destin de l’Amérique qui imposerait la démocratie au Vieux Continent fragilisé par la guerre pour le bien-être de l'humanité en voie d'unification.

Si Tocqueville s'est manifestement trompé dans ses descriptions de l'Amérique, c'est bien parce que ses intentions étaient ailleurs. En effet, d'après Carl Schmitt, La Démocratie en Amérique était moins une étude socio-politique qu'une entreprise d'édification. «Il était en Amérique et écrivait à propos de l'Amérique. But his heart was in France and the thought of France, never absent from him, 
unconsciously colored every picture he drew» (ibid., p. Io8). En livrant un tableau de la démocratie en tant que telle plutôt qu'une juste perception de l'Amérique, Tocqueville aurait, avant tout, cherché à mettre la France en garde contre les dangers du phénomène démocratique qu’il avait cernés avec clairvoyance. Contrairement à la plupart des historiens, il n'avait pas sombré dans l'historicisme de son siècle, pour la simple et bonne raison qu'il avait été vaincu. L'auteur de La Notion de politique pensait que la connaissance historique fondamentale n'était accessible qu'à l'homme qui avait connu l'expérience de la défaite ${ }^{14}$. Schmitt (2003 [1950], p. I38) aime à remarquer que Tocqueville «appartenait à la couche sociale qui avait été vaincue par la Révolution française de I789».

Dans Parlementarisme et démocratie (1988, [1923], p. 29), le juriste allemand relève, en évoquant Tocqueville, qu'en France, tout portait à croire au XIX ${ }^{e}$ siècle qu'«il n’y avait apparemment plus de digue depuis I789 "contre” la marée de la démocratie ». Au moyen de ses descriptions biaisées, Tocqueville aurait pourtant entrepris d'exhorter les Français à retrouver le terreau national et l'ancien modèle d’organisation sociale démantelé suite aux événements de i789. Cette entreprise d'édification, ce projet d'un retour à la situation pré-révolutionnaire, prenait donc appui sur un sérieux avertissement, «un avertissement adressé à la France, afin qu'elle se procure une base morale et religieuse pour sa vie nationale et qu'elle reconstruise l'édifice social que la Révolution avait renversé » (dans Tommissen, 200I, p. I08). À en croire Carl Schmitt, c'était bien là que se situait le cœur des deux tomes que l'aristocrate consacra à l'étude de l'Amérique. Tocqueville apparaît ainsi sous les traits d'un penseur contre-révolutionnaire. Dans sa critique de l'universalisme politique, le juriste conservateur semble s'inscrire de façon quasi explicite dans le sillage de Tocqueville, du moins dans le sillage de Tocqueville tel qu'il l’avait compris. L’aversion du penseur français contre les valeurs inhérentes à la révolution de i789, qui seraient à l'origine de la centralisation et de la démocratisation qu’il pronostiqua en I835, constituerait une sorte de plaidoyer pour la pluralité du monde, pour un pluriversum d'entités reconverties à la doctrine de la vie nationale. La compréhension schmit-

14 À ce sujet, voir Schmitt (2003 [1950], p. 138), qui semble, apparemment, s'identifier à l'auteur du XIX siècle sur ce point. Arrêté et emprisonné en 1945, il ferait lui aussi partie du camp des vaincus. 
tienne de la guerre froide paraît, par conséquent, s'abreuver à la source de la fameuse prédiction de Tocqueville et des considérations normatives qui accompagneraient une telle prévision - la critique de l'Empire évoque sensiblement l'opposition déterminée de Schmitt à l'unification du monde en devenir que consignerait la «pax américano-soviétique». En outre, dans les raisonnements du juriste, la garantie d'un «plurivers » découle ouvertement de la coexistence d'entités nationales homogènes, acquises aux vertus de l'égalité démocratique.

Si Schmitt recourt aux dires de Tocqueville pour critiquer l'homogénéité croissante de l'humanité, il se peut qu'il transforme les mots de l'historien. En effet, le «plurivers», quoique Tocqueville n’utilise évidemment pas ce terme, est nécessaire à la survie de la diversité et de la liberté, ainsi qu’à la protection de l'équilibre démocratique per se. En 1944, Schmitt écrit:

Pour Tocqueville, la révolution de 1789 est le symptôme d'un processus de centralisation irrésistible, qui se sert de toutes les formes d'État, de tous les partis, de toutes les idéologies et se poursuit irrémédiablement. La Révolution Française recommencera toujours et c'est toujours la même. (1950a, p. 90)

Remise dans le contexte original des Souvenirs, soit les événements de I848 en France, cette dernière phrase fait référence aux crises qui ont suivi la Révolution française; elle ne peut être considérée comme une critique de celle-ci¹5. Il semblerait alors que le philosophe allemand ne comprenne pas avec justesse les propos du théoricien français. Schmitt considère que l'aristocrate était de facto un vaincu de la Révolution et donc un auteur particulièrement critique de cette dernière. Tocqueville était cependant conscient que la Révolution était inévitable ou «providentielle» (1986 [I835], p. 4I) selon ses mots, et qu'il était dès lors inutile de regretter l'Ancien Régime. Il semble tout aussi clair que ce qu’il «redoute le plus pour les générations à venir, ce ne sont pas les révolutions », mais l’immobilisme d’une société dépourvue d’un tel moyen pour mettre en place des idées nouvelles (198I [I840], p. 323).

15 Tocqueville (1999 [1964], p. 89) dit: «Après chacune de ces mutations successives, on avait dit que la Révolution française ayant achevé ce qu'on appelait présomptueusement son œuvre était finie: on l'avait dit et on l'avait cru. Hélas! je l'avais espéré moi-même sous la restauration et encore, depuis que le gouvernement de la restauration fut tombé. Et voici la Révolution française qui recommence, car c'est toujours la même». 


\section{UNE CRITIQUE PARTAGÉE DE L'INDIVIDUALISME ET DE LA SOUVERAINETÉ POPULAIRE}

Les tendances à l'ingérence et à la criminalisation de l'agresseur accompagneraient inévitablement l'avènement de l'ère démocratique. «Naturellement, affirme Schmitt, aucun système démocratique ne peut échapper à cette chute originelle. La plate-forme de l'égalité démocratique n'est que la planche pour sauter dans une nouvelle inégalité. C'est là l’angoisse personnelle de Tocqueville » (I99I, p. 240). L'égalité «libérale-démocratique» viendrait mettre à mal la véritable égalité, celle qui concerne les nationaux et qui devrait caractériser la démocratie. En tentant de donner un contenu à cette dernière, la bourgeoisie libérale chercha à la déplacer du terrain politique vers le terrain économique. «En vérité, on opérait là une transformation essentielle de la notion de démocratie, car il est impossible de transférer des points de vue politiques sur des relations économiques tant que règnent, en économie, la liberté contractuelle et le droit privé » (Schmitt, 1988 [1923], p. 30). Dès l'instant où elle est édifiée sur la base du droit privé, la démocratie cesse d'être politique, puisque dorénavant la forme qu'elle représente diffère lourdement du contenu de l'autorité et de la publicité.

Les groupes socio-économiques qui entendent s'organiser «démocratiquement» ne sauraient composer un seul et même sujet que d'une façon éminemment abstraite. Concrètement, il s'agit bien de masses essentiellement hétérogènes. Dans La Notion de politique, Schmitt (2004 [1932], p. I14) se pose la question «de savoir si le principe pur et rigoureux du libéralisme individualiste peut donner naissance à une conception spécifiquement politique ». Il y répond, évidemment, par la négative. Aussi, l'État de droit bourgeois qui assure les libertés individuelles consignerait l'existence d'un peuple pluriel et endosserait, de ce fait, les pourtours de l'anti-démocratie. La reconnaissance du primat de l'individu, consubstantielle au libéralisme, transformerait les citoyens en de simples consommateurs. En affirmant la préséance des droits individuels, la doctrine libérale briserait l'homogénéité de l'amitié nationale: chaque individu se dissocierait de la nation au motif de ses intérêts propres (que ceux-ci soient économiques ou non). 
Schmitt a bien vu que Tocqueville énonçait le même constat au XIX ${ }^{e}$ siècle. La critique tocquevillienne de l'individualisme est tout aussi virulente que celle du juriste westphalien: «Laristocratie avait fait de tous les citoyens une longue chaîne qui remontait du paysan au roi; la démocratie brise la chaîne et met chaque anneau à part» (Tocqueville, 198I [1840], p. 127). Cette absence de hiérarchie a toutefois une autre signification pour Tocqueville. Dorénavant, les individus adopteraient certains points de vue parce qu'ils ne possèdent plus de place déterminée dans la société. En se soumettant à l'omnipotence de la majorité, au pouvoir corrompu de la souveraineté populaire, l'individu s'asservirait et perdrait toute envie et toute possibilité de participer à la vie politique; il en viendrait même à «renoncer à ses droits de citoyen, et pour ainsi dire à sa qualité d'homme» (Tocqueville, I986 [1835], p. 385). En outre, une société tournée vers la recherche effrénée du bien-être matériel, une société de consommateurs comme l'écrirait Schmitt, serait, pour Tocqueville, une société qu'il faut craindre, une société où le matérialisme a «amolli» les individus (Tocqueville, 1981 [1840], p. 167). Même si cela peut avoir des conséquences dramatiques sur l'organisation politique de la société, cette isolation grandissante ne signifie pas forcément qu'ils sont politiquement isolés. Au travers des fameux concepts de participation politique et de société civile, le théoricien français croit que les individus peuvent agir en tant que groupe, en particulier grâce à leurs appartenances à des associations, ce qui n'est pas sans rappeler sa conception ambiguë de l'homogénéité. Par ailleurs, là où Schmitt blâme la liberté, Tocqueville blâme l'égalité, qui serait, selon lui, responsable de cette désolidarisation de la société (Tocqueville, 1986 [1835], p. 389), et il compte sur l'«intérêt bien entendu» pour contrebalancer cette égalité excessive. Cet intérêt, concept étranger à Schmitt, implique que les individus combinent «leur propre bien-être avec celui de leurs concitoyens » au moyen d'un intérêt éclairé qui est, «de toutes les théories philosophiques, la mieux appropriée aux besoins des hommes de notre temps», entendons de l'homme démocratique (Tocqueville, 198I [1840], p. I53-54). 
Ainsi, Tocqueville et Schmitt se retrouvent dans une critique partagée de l'individualisme - le juriste de Weimar en était d’ailleurs pleinement conscient, lorsqu'il évoquait «l'angoisse personnelle » du grand historien français -, mais leurs divergences normatives au sujet du sens et, surtout, de la place à attribuer à l'égalité dans le dispositif démocratique constituent une sérieuse pierre d'achoppement. Comme l'argumente le théoricien français, son concept de démocratie a besoin à la fois de la liberté et de l'égalité pour être maintenu : «Les hommes seront parfaitement libres, parce qu'ils seront tous entièrement égaux; et ils seront tous parfaitement égaux parce qu'ils seront entièrement libres » (ibid., p. I19) ${ }^{16}$. D’autre part, Carl Schmitt s'emploie à démontrer que la démocratie véritable n'est pas d'essence libérale, tant elle s'oppose à l'alternative théorique qui conduirait à l'atomisation inévitable du peuple homogène. Même si Tocqueville a discerné les dangers que charriait l'individualisme démocratique, sans doute n’a-t-il pas vu, estime Schmitt, qu’en accordant une place prépondérante à la «liberté», il déstabilisait l'effectivité de la représentation; autrement dit, le processus d'identification caractéristique des régimes démocratiques authentiques qui devrait tendre à l'identité parfaite entre les gouvernés et les gouvernants.

Afin de permettre la représentation effective du peuple, l'égalité démocratique ne saurait souffrir aucune restriction de nature libérale. Seul un peuple qui existe en tant qu'unité politique peut être représenté, c'est-à-dire rendu présent sur la scène publique par la médiation d'un être - d'un représentant en l'occurrence - qui est, pour sa part, empiriquement présent. Cette idée de représentation, essentielle à la démocratie telle que l'entend Schmitt, requiert dès lors l'aptitude à faire fi des intérêts individuels qui dissoudraient le «bien commun » de la nation. Comprenons qu’un intérêt privé ou particulier ne peut aucunement faire l'objet d'une représentation. Si la notion d'égalité est mise au service de la liberté, la constitution d'un peuple comme sujet du pouvoir constituant est vouée à l'échec. Dans ses notes posthumes, Carl Schmitt estime que Tocqueville «has failed to grasp the substantial identity of the American people» (dans Tommissen p. ı०8), conséquence évidente de son libéralisme. 
Tocqueville adopte en effet une conception plutôt libérale de la notion d'identité politique. Dans une des lettres qu'il adresse à Arthur de Gobineau, il rejette toute possibilité de déterminisme, que celui-ci soit social, géographique ou historique, au profit de l'inviolabilité de la liberté humaine (Tocqueville, I959, p. 202). Pourtant, il est surprenant de constater que Schmitt ne semble pas avoir vu que Tocqueville reconnaissait des limites au libéralisme. Si Tocqueville n’a pas perçu la substance de l'identité américaine, c'est pour la simple et bonne raison que, suivant l'historien français, une telle identité n'existait pas encore. La Fédération américaine prenait appui à l'époque sur l'intérêt des États qu'elle réunissait et s’avérait par conséquent instable. L’auteur français écrit : «Les habitants des États-Unis parlent beaucoup de leur amour pour la patrie ; j’avoue que je ne me fie point à ce patriotisme réfléchi qui se fonde sur l'intérêt, et que l'intérêt, en changeant d’objet, peut détruire » (Tocqueville, I986 [1835], p. 543). Les intérêts peuvent changer, évoluer voire disparaître. Tocqueville met donc ses lecteurs en garde contre les associations qui rassemblent un trop grand nombre d'intérêts divergents, et donc contre les États bien trop hétérogènes. Oui, l'auteur était un libéral et n’adoptait guère une conception strictement substantielle de l'identité ; mais il pose néanmoins certaines bornes au libéralisme. La providence «trace, il est vrai, autour de chaque homme, un cercle fatal, dont il ne peut sortir; mais dans ses vastes limites, l'homme est puissant et libre; ainsi des peuples» (Tocqueville, 198I [1840], p. 402). Ce cercle apparaît également comme la garantie de la liberté qui peut prospérer, liant indéniablement la survie de cette dernière à la survie du politique. Le Français ne pouvait pas imaginer la liberté en dehors d'un corps politique prédéterminé suffisamment unifié en vue d'offrir à sa population les motifs d'une participation à la vie politique et de maintenir ainsi en vie ce qui les lie politiquement.

Le juriste conservateur conteste l'hypothèse selon laquelle, si le monde était centralisé et l'humanité complètement démocratisée, «un gouvernement de l'homme par l'homme sera[it] superflu parce que les hommes [...] [seraient] alors totalement libres» (Schmitt, 2004 [1932], p. I00-IOI). La question est alors de savoir ce que les hommes feraient de cette liberté. Le peuple a plutôt besoin d'être représenté pour que persiste l'état civil, sans quoi, il risquerait 
de succomber à la menace d'une rechute dans l'état de nature. Si l'auteur allemand affirme ouvertement que tout pouvoir prend sa source dans l'existence préalable d'un peuple capable d'action, ce dernier n'est pas à même de décider.

En dernier ressort, le peuple n'est pas souverain au sens strict du terme. Plus exactement, le pouvoir vient du peuple, au sens où celui-ci exprime la voluntas que le représentant rationalisera politiquement au moyen de décisions. Bien que Schmitt semble contester l'idée de la «tyrannie égalitaire», force est de reconnaître qu'il existe une certaine ambivalence chez le juriste, qui craignait plus que tout la tyrannie du peuple, arguant une version limitée de la souveraineté populaire. Selon lui, le peuple est mauvais par nature, il est frappé du sceau de la corruption adamique originelle, d'où la nécessité du gouvernement et la relativisation immédiate du concept de souveraineté populaire. Déjà en I92I, dans Die Diktatur, le juriste soulignait que «la méchanceté naturelle de l'homme est un axiome » (Schmitt, 2000 [192I], p. 28) et que la dangerosité du peuple se doit d'être domestiquée. L’assertion d'un pessimisme anthropologique constitue le point de départ de ses raisonnements politico-philosophiques de jeunesse, puisque, à l'en croire, «toutes les théories politiques véritables postulent un homme corrompu, c'est-à-dire un être dangereux et dynamique, parfaitement problématique » (Schmitt, 2004 [1932], p. I05). Dans La Notion de politique, Schmitt conclut son chapitre consacré aux fondements anthropologiques des théories politiques en se référant au second tome de La Démocratie en Amérique. «Avant la Révolution de I789, la société aristocratique française était éprise de l’homme bon de nature et du peuple vertueux et si touchant» (ibid., p. II3). L'historien français avait décrit cette situation des plus étranges avec l'éloquence et le sens politique qui le caractérisent : à l'époque, personne n’avait senti que la révolution se profilait à l'horizon. Carl Schmitt s'étonne avec Tocqueville de voir «la sécurité et l'aveuglement de ces privilégiés qui parlent de la bonté, de la douceur et de l'innocence du peuple quand déjà 93 est sous leurs pieds, spectacle ridicule et terrible » (ibid., p. I I4). Reprenant les mots de Tocqueville, le juriste sous-entend que le penseur français partageait, lui aussi, cette croyance en la dangerosité du peuple. La convergence entre nos deux auteurs sur ce sujet s'arrête pourtant à cet endroit. Malgré les indéniables correspondances, le juriste allemand ne se 
réfère à Tocqueville quau terme du chapitre dont il est ici question, prenant bien soin d'éluder le nom du grand Français lorsqu'il évoque la thématique du péché originel chez les penseurs contre-révolutionnaires. En tant que chrétien, «ce qu’il [Tocqueville] est resté suivant la croyance de ses pères reçue du baptême et de la tradition, il a succombé à l'agnosticisme scientifique de son époque» (Schmitt, 2003 [1950], p. 138). Schmitt estime que, pour cette raison, Tocqueville na pas su percevoir que le continent européen, déclassé par l’Amérique et la Russie, pouvait recouvrir son lustre d’antan s'il était envisagé à partir de la figure néo-testamentaire du katechon, qui chez saint Paul est la force qui retient la violence de l'Antéchrist et qui garantit la pérennité de l'histoire. En désignant l'ennemi du «grand espace [Großraum]», le centre impérial assurerait le mouvement politico-dialectique de l'histoire. Il en certifierait la durabilité et retarderait l'avènement d'une unification du monde qu'entreprendrait la puissance américaine libérale, identifiée à l’Antéchrist. L’universalisme libéral recourrait à tous les moyens techniques possibles en vue de pourchasser l'adversaire de l'amitié universelle, mettant la terre à feu et à sang et précipitant du même geste la fin de l'éon présent. Tocqueville ne connaissait aucun katechon, il lui «manquait le soutien de l'histoire sacrée pour préserver du désespoir son idée historique de l'Europe » (ibid.). Il était certes catholique, affirme Carl Schmitt (2008 [1923], p. 7), mais son libéralisme l'empêcha de voir dans cette doctrine politique, portée par les Américains, l'incarnation historique de l'Antéchrist" ${ }^{17}$.

André Doremus (dans Schmitt, 2003 [1950], p. 224) soutient qu'«une lecture rapide du texte de Tocqueville peut donner l'impression que Schmitt ne lui rend pas entièrement justice en disant qu'il n'avait pas de katechon ». Tocqueville pointe en effet certains facteurs qui freineraient le développement d'une humanité mondiale. On peut aussi penser que Schmitt n’interprète pas Tocqueville correctement lorsqu'il affirme que ce dernier est effrayé par le peuple. Dans sa Démocratie en Amérique, l’aristocrate craint le pouvoir de la masse et tout particulièrement la tyrannie de la majorité qui en découle (Tocqueville, I986 [1835], p. 369-387), mais il a confiance en l'individu.

17 Sur l'importance des notions théologique de katechon et d'Antéchrist dans la pensée de Carl Schmitt, voir Storme (2008, p. 163-225). 
James Schleifer (2000, p. 80) a très bien rapporté cet argument. Il montre que l'écrivain français pensait, de prime abord, que les lois étaient la principale cause des actions humaines; mais Tocqueville aurait modifié cette première impression concernant le peuple américain, après avoir réalisé qu’une telle perspective laissait trop peu de place à la volonté des individus. En dépit de cela, Schmitt semble retenir que, pour l'auteur du XIX ${ }^{\mathrm{e}}$ siècle, le peuple qui avance sur les rails de l'embourgeoisement ne serait qu'un «troupeau d'animaux industrieux et timides» (dans Schmitt, 1988 [1923], p. 29). Il paraît confirmer la sombre interprétation tocquevillienne de l'âge démocratique auquel il tentera de répondre, dans un premier temps, par sa théorie de l'État total qui apparaît tel «un moyen pour arrêter l'évolution lente et sûre vers cette sorte d’État que Tocqueville [...] désignait sous le terme de "despotisme démocratique" et à laquelle il associait la centralisation administrative » (Campagna, 2004, p. 32 et Scheuerman, 1995, p. 86-87) et la tyrannie de la majorité. D’un autre côté, Tocqueville préconisait un retour du citoyen dans la sphère politique, dans ce monde duquel il s’est éloigné car «la vie n’est ni un plaisir, ni une douleur, c'est une affaire grave dont nous sommes chargés et qu'il faut conduire et terminer à notre honneur » (Tocqueville, I99I, p. I7I). Ces mots ne sont pas sans remémorer ouvertement l'«existentialisme politique» de Carl Schmitt qui voyait dans les doctrines jugées apolitiques, voire anti-politiques, un manquement au sérieux de l'existence que l'on ne saurait assumer qu'à travers le concept de politique (Wolin, 1990).

\section{CONCLUSION}

Un cadre d'analyse théologico-politique?

Par l'intermédiaire de questions princeps à première vue opposées, situées l'une et l'autre aux deux pôles extrêmes d'un problème fondamental de la théorie politique, Schmitt et Tocqueville répondent, chacun à leur manière, à l'interrogation de savoir si le phénomène démocratique ne provoquerait pas une autodissolution dialectique du politique. Il existerait un point par lequel les questions centrales des deux auteurs peuvent se transvaser l'une dans l'autre, que l'on peut identifier comme étant la crainte des «dépolitisations». Si l’historien français s’inquiète de 
préserver la liberté dans un contexte d'égalité, il n'omet pas pour autant de souligner que la sauvegarde de la liberté passerait néanmoins par la préservation de la politique. Les deux questions princeps hétérogènes avancées dans notre introduction admettent ainsi une formulation commune que l'on pourrait résumer comme suit: comment éviter la réduction du citoyen au consommateur? Comment sauver la dimension politique de l'existence? Parlant depuis des lieux et des contextes théoriques divergents, les deux auteurs diagnostiquent les difficultés du politique à se maintenir effectif en régime démocratique; ils cherchent à sauver ce qui peut l'être, développant une sorte de sotériologie politique partagée qui a pris conscience, à des époques différentes, du risque d'une dégradation consumériste du citoyen. Même s'il tord à souhait les réflexions du grand Français, Carl Schmitt avait manifestement saisi cette profonde convergence problématique qui le liait au «plus grand historien du XIX siècle». Dans cette conclusion, nous voudrions faire l'hypothèse que ce cadre d'analyse commun peut s'expliquer à partir d'enseignements d'ordre théologico-politique, inspirés par un certain augustinisme décelable dans la pensée des deux auteurs. Leur approche de la société, en tant que continuation d'une époque pré-moderne, est construite sur la nécessité d’un système de nature religieuse (civile ou non) capable de soutenir l'existence du politique.

«Tous les concepts prégnants de la théorie moderne de l'État sont des concepts théologiques sécularisés» (Schmitt, 1988 [1922], p.46). Pour le juriste westphalien, la conceptualité politique moderne a hérité des structures de la sphère théologico-religieuse. L'époque moderne ne saurait s’affirmer d'elle-même à partir de ses ressources propres - d'un point de vue structurel, elle dépend indéniablement de la conceptualité théologique ${ }^{18}$. Quoique la lecture schmittienne de Tocqueville indique quaux yeux du juriste, les droits libéraux constituent un solide héritage issu des révolutions européennes des $\mathrm{XVIII}^{\mathrm{e}}$ et $\mathrm{XIX}$ siècles, les catégories déterminantes du droit public (Staatsrecht) seraient entièrement redevables aux notions tirées de l'ère pré-moderne, pilotée quant à elle par des idées théologiques et métaphysiques. Lorsqu'il s'inquiète de l'immanentisme de Krabbe ou de Kelsen, le juriste allemand se réfère ainsi à Tocqueville, qui naurait pas renié l’origine métaphysique de la sphère du politique: 
Dans son tableau de la démocratie américaine, Tocqueville déclarait encore que dans l'idée démocratique le peuple planait au-dessus de la totalité de la vie étatique comme Dieu au-dessus du monde : comme origine et fin de toutes choses, comme ce dont tout sort et où tout revient (ibid., p.58).

Suite à l'apparition de l'ennemi, les individus qui se sentent existentiellement menacés se retranchent derrière la protection de l'État, capable de les défendre et de domestiquer leur nature corrompue. Carl Schmitt se situe, en réalité, aux rives d'un augustinisme radical de tradition luthérienne ou pascalienne. Parce que l'homme est mauvais par nature, il a besoin d'être gouverné; si l'autorité séculière brandit le glaive, c'est bien en raison de la nature pervertie des individus. Le péché adamique est à l'origine de l'État (sans lequel les êtres humains se déchireraient dans l'état de nature), thème augustinien par excellence. Comme l'aurait affirmé l'Évêque d'Hippone, la forme étatique représente le remedium peccati - le remède au sombre destin de l'humanité.

Dans une lettre qu'il adresse à son cousin et confident Louis de Kergolay le ı novembre i836, Tocqueville énonce ses trois sources principales d'inspiration. «Il y a trois hommes avec lesquels je vis tous les jours un peu, c'est Pascal, Montesquieu et Rousseau» (Tocqueville, 1977a). Schmitt a raison de souligner le rapprochement que Tocqueville effectue quand il compare le peuple à Dieu dans son chapitre consacré à la souveraineté populaire. De plus, son recours au concept de Providence, alors qu’il évoque l'inévitabilité de l'avènement de la démocratie, ne semble nullement innocent. En procédant de la sorte, l’auteur français saccorde avec la thèse de Schmitt selon laquelle la modernité ne serait pas le fruit de changements majeurs mais plutôt la continuité ou le prolongement de l'époque pré-moderne. «Tout ce que la Révolution a fait, se fût fait, je n’en doute pas, sans elle; elle n’a été qu’un procédé violent et rapide à l'aide duquel on a adapté l'état politique à l'état social, les faits aux idées, les lois aux mœurs » (Tocqueville, 1988 [1856], p. 85). Des auteurs tels que Joshua Mitchell (1995) ou Lucien Jaume (2008, p. 94) ont exploré cet aspect théologique de la pensée tocquevillienne. Alors que Mitchell relie clairement l'auteur français à la pensée d’Augustin, à travers une interprétation qu'ils partageraient d'un homme irrationnel, qui erre entre deux versions extrêmes de la vie (l'une repliée sur elle-même, l'autre rétive), Jaume nous offre une analyse lexicographique 
ingénieuse de l'œuvre de Tocqueville. Il se concentre tout particulièrement sur la résurgence du mot «dépôt» en association avec la notion d’autorité démocratique. Tocqueville utilise le mot à trois reprises et toujours en référence à la religion, évoquant de la sorte l'émergence d'une nouvelle religion civile. Le commentateur français soutient ainsi que l'historien du XIX siècle usait d'un vocabulaire théologico-religieux pour renforcer le fait que le monde démocratique, en particulier l'opinion publique qu'il recèle, serait un Dieu nouveau, une religion nouvelle. Bien que l'augustinisme de Tocqueville soit sujet à débats, son recours à un lexique religieux, ainsi que sa croyance en la Providence, témoignent de ce qu'il inscrivait ses observations dans une perspective de nature théologique.

Étant donné que, pour nos deux penseurs, la forme étatique-démocratique est d'ordre providentiel et que la conceptualité post-I789 paraît conserver l'essentiel de la systématicité théologique (le peuple agissant comme Dieu, du roi au peuple), il ne serait pas inconséquent d'émettre l'hypothèse que leurs préhensions respectives de la démocratie partagent un même tracé discursif. Toujours empreints de leurs traces et de leur «structuralité» originelle, les concepts qui participent de la définition du phénomène démocratique ne peuvent sans doute, chez Schmitt et Tocqueville, que s'agencer de façons plus ou moins correspondantes. Mais nous ne devons toutefois pas perdre de vue que le 5 août I836, Alexis de Tocqueville (1977a) confiait à Louis de Kergolay que l'homme n'est ni bon ni mauvais et que son devoir est de trouver un juste milieu entre ces deux extrêmes; il soulignait de nouveau la foi qu'il place dans les individus et leur libre-arbitre, confirmant par là même l'insurmontable différence qui le sépare de Carl Schmitt.

\section{BIBLIOGRAPHIE}

AGNOLI J. et BRÜCKNER P., 1968, Die Transformation der Demokratie, Francfort-sur-le-Main, Europäische Verlagsanstalt.

ANTOINE A., 2003, L'Impensé de la démocratie. Tocqueville, la citoyenneté et la religion, Paris, Fayard. 
AUDIER S., 2004, Tocqueville retrouvé. Genèse et enjeux du renouveau tocquevillien français, Paris, Vrin-EHESS.

AUDIER S., 2009, La Pensée anti-68. Essai sur une restauration intellectuelle, Paris, La Découverte.

BALAKRISHNAN G., 2006 [2000], L'Ennemi. Un portrait intellectuel de Carl Schmitt, Paris, Éd. Amsterdam.

BLUMENBERG H., 1999, La Légitimité des Temps modernes, Paris, Gallimard.

CAMPAGNA N., 2004, Le Droit, le Politique et la Guerre. Deux chapitres sur la doctrine de Carl Schmitt, Laval, Presses de I'Université Laval.

CLINTON D., 2003, Tocqueville, Lieber, and Bagehot: Liberalism confronts the World, New York, Palgrave Macmillan.

DERRIDA J., 1994, Politiques de l'amitié, Paris, Galilée.

JACQUES D.« «Tocqueville et le problème de la clôture politique», dans: Guellec, L., (éd.), 2005, Tocqueville et l'esprit de la démocratie, Paris, Presses de Sciences Po.

JANET P., 1887, Histoire de la science politique dans ses rapports avec la morale, vol. 2, Paris, Félix Alcan.

JAUME L., 2008, Tocqueville. Les Sources aristocratiques de la liberté, Paris, Fayard.

KAHAN A. S., 1992, Aristocratic Liberalism: the Social and Political Thought of Jacob Burckhardt, John Stuart Mill and Alexis de Tocqueville, Oxford, Oxford University Press.

KALYVAS A. et KATZNELSON I., 2008, Liberal Beginnings: Making a Republic for the Moderns, Cambridge, Cambridge University Press.

LINDENBERG D., 2002, Le Rappel à l'ordre. Enquête sur les nouveaux réactionnaires, Paris, Seuil.

MANENT P., 2006, La Raison des Nations. Réflexions sur la démocratie en Europe, Paris, Gallimard.

MEUWLY O., 2002, Liberté et Société. Constant et Tocqueville face aux limites du libéralisme moderne, Genève, Librairie Droz.

MITCHELL J., 1995, The Fragility of Freedom: Tocqueville on Religion, Democracy, and the American Future, Chicago, University of Chicago Press. 
NORRIS A., 1998, «Carl Schmitt on friends, enemies and the political», Telos, n० II2, été. OSSEWAARDE M., 2008, «Tocqueville and the Continuation of the TheologicalPolitical», European Journal of Political Theory, vol. 7, n I.

PITTS J., 2005, A Turn to Empire: The Rise of Imperial Liberalism in Britain and France, Princeton, Princeton University Press.

ROHRMOSER G., 1998, «Der Hegelsche Staat ist tot», dans: Tommissen, P., (éd.), Schmittiana. Beiträge zu Leben und Werk Carl Schmitts, vol. 6, Berlin, Duncker \& Humblot.

SCHEUERMAN W., 1995, «ls parliamentarism in crisis? A response to Carl Schmitt», Theory and Society, vol. 24, n I.

-, 1999, Carl Schmitt: The End of Law, New York/ Oxford, Rowman \& Littlefield Publishers.

SCHLEIFER J., 2000, The Making of Tocqueville's Democracy in America, Indianapolis, Liberty Fund Inc.

SCHMITT C., 1949, «Commento a Tocqueville. L'idea dei diritti», Universitas, vol. $4, n^{\circ}$ 5, p. 587-588.

-, 1950, Ex Captivitate Salus. Erfahrungen der Zeit 1945-1947, Cologne, Greven Verlag.

-, 1950a, Donoso Cortés in gesamteuropäischer Interpretation. Vier Aufsätze, Cologne, Greven Verlag.

-, 1988 [1922], Théologie politique, Paris, Gallimard.

-, 1988 [1923], Parlementarisme et démocratie, Paris, Seuil.

-, 1990, «L'État de droit bourgeois », dans : Schmitt, C., Du Politique. « Légalité et légitimité » et autre essais, Puiseaux, Pardès.

-, 1991, Glossarium. Aufzeichnungen der Jahre 1947-1951, Berlin, Duncker \& Humblot.

-, 1993 [1928], Théorie de la Constitution, Paris, PUF.

-, 2000 [1921], La Dictature, Paris, Seuil.

-, 2003 [1950], Ex Captivitate Salus. Expériences des années 1945-1947, Paris, Vrin.

-, 2004 [1932], La Notion de politique, Paris, Flammarion.

-, 2004a [1932], « L’Ère des neutralisations et des dépolitisations », dans Schmitt, La Notion de politique, Paris, Flammarion. 
-, 2005 [1927 ], Frieden oder Pazifismus? Arbeiten zum Völkerrecht und zur internationalen Politik 1924-1978, Berlin, Duncker \& Humblot.

-, 2008 [1923], Römischer Katholizismus und politische Form, Stuttgart, Klett-Cotta.

STORME T., 2008, Carl Schmitt et le marcionisme. L'impossibilité théologico-politique d'un œcuménisme judéo-chrétien?, Paris, Cerf.

TERTULIAN N., 1996, «Carl Schmitt entre catholicisme et national-socialisme», Les Temps Modernes, $\mathrm{n}^{\circ} 594$.

TOCQUEVILLE A. (de), 1959, Euvres complètes, vol. 9, Paris, Gallimard.

-, 1973, Tocqueville au Bas-Canada. Écrits datant de 1831 à 1859. Datant de son voyage en Amérique et après son retour en Europe, Montréal, Éditions du Jour.

-, 1977, Égalité sociale et liberté politique, Paris, Aubier-Montaigne.

-, 1977a, Euvres complètes, vol. 7, Paris, Gallimard.

-, 1981 [1840], De la Démocratie en Amérique, vol. 2, Paris, Flammarion.

-, 1986 [1835], De la Démocratie en Amérique, vol. I, Paris, Gallimard.

-, 1988 [1856], L'Ancien Régime et la Révolution, Paris, Flammarion.

-, 1991, Euvres, vol. I, Paris, Gallimard.

-, 1999 [1964], Souvenirs, Paris, Gallimard.

TOMMISSEN P., (éd.), 200I, Schmittiana. Beiträge zu Leben und Werk Carl Schmitts, vol. 7, Berlin: Duncker \& Humblot.

WOLIN R., 1990, «Carl Schmitt, political existentialism, and the total State», Theory and Society, vol. 19, n4.

WOLIN S., 200I, Tocqueville between two Worlds: The Making of a Political and Theoretical Life, Princeton, Princeton University Press. 